\title{
CdTe Photo-Luminescent Quantum Dots as a Possible New Method to Improve Solar Cell Efficiency.
}

\author{
A. Zazueta-Raynaud ${ }^{1,2}$, A. Flores-Pacheco ${ }^{2}$, G. Saavedra-Rodríguez ${ }^{2}$, J. E. Pelayo ${ }^{1,3}$, R. Lopez- \\ Delgado $^{1,2}$, M. E. Álvarez-Ramos ${ }^{2}$ and A. Ayon ${ }^{1}$ \\ 1. University of Texas at San Antonio, Dept. of Physics and Astronomy, MEMS Research Lab, One \\ UTSA Circle, San Antonio, TX 78249, USA. \\ 2. Universidad de Sonora, Departamento de Física, Blvd. Luis Encinas y Rosales, Col. Centro, \\ Hermosillo, Sonora, México. CP 83000. \\ 3. Universidad de Guadalajara, Centro de Ciencias Exactas e Ingenierias, Blvd. Gral. Marcelino García \\ Barragán 1421, Olímpica, 44430 Guadalajara, Jalisco, México.
}

In terms of cost and power conversion efficiency solar cells are considered to be the most promising renewable energy source for the foreseeable future. Additionally, they are pollution free. However, on theoretical grounds, there is room for improvement both in the manufacturing cost and the efficiency of the marketed devices. Indeed, solar cell technology must be developed and improved further to obtain optimal efficiency and cost. As far as the materials employed are concerned, silicon solar cells are the preferred market choice and, for the same reason, have captured more than eighty percent of the current photovoltaic market due to their relatively high efficiency in spite of their relatively high manufacturing cost [1]. On the other hand, the relatively poor UV absorption of crystalline silicon (c-Si) limits the power conversion efficiency that can be attained precluding their proliferation in a larger number of settings. Higher efficiency is, therefore, the greatest challenge for their expanded utilization.

In order to address the aforementioned shortcomings of currently employed silicon solar cells, we describe the synthesis, characterization and incorporation on photovoltaic structures of photoluminescent Cadmium Telluride (CdTe) quantum dots (QDs) that exhibited a size-dependent Stokes shift that could enable dramatic improvements in the power conversion efficiency [2]. To this end, CdTe quantum dots were synthesized, cleaned, collected by centrifugation to promote their precipitation, and dispersed in Poly methyl methacrylate (PMMA) as a matrix for de CdTe quantum dots and deployed on soda lime substrates to study their optical properties.

The excitation wavelengths for the CdTe QDs ranged from 280 to $450 \mathrm{~nm}$ and the observed peak emissions ranged from 545 to $623 \mathrm{~nm}$, contingent on the synthesis time. Ostensibly, CdTe QDs enable the down conversion of high energy photons to photons more suitable for electron-hole pair production in Si-based solar cells [3].

The colloidal CdTe quantum dots were synthesized in a water-based solution with the ultimate size being determined by the synthesis time employed. Since the size determines the emission wavelength, evidently it can be tuned by adjusting the chemical reaction time [4]. The characterization effort comprised SEM inspection, photoluminescence (PL), absorption and transmittance. QD size, band gap energy and quantum confinement were calculated employing Brus', Tauc's and Bohr's radius equations. The observations indicate that the judicious utilization of the down shifting effect exhibited by Cadmium Telluride quantum dots have the potential to improve the power conversion efficiency of photovoltaic devices. 
References:

[1] Renata Reisfeld, Optical Materials 32 (2010), p. 850.

[2] Suli Wu, et al, Journal of Materials Chemistry 22 (2012), p. 14573.

[3] Keith R. McIntosh, et al, Progress in Photovoltaics: Research \& Applications (2009) 17, p. 191.

[4] B.S. Richards, Solar Energy Materials \& Solar Cells 90 (2006), p. 1189.

[5] The authors gratefully acknowledges the founding provided by CONACyT (Mexico), the project number FESEM JEOL JSM-7800F and the U.S. Army Research Office (Grant W911NF-13-1-0110).
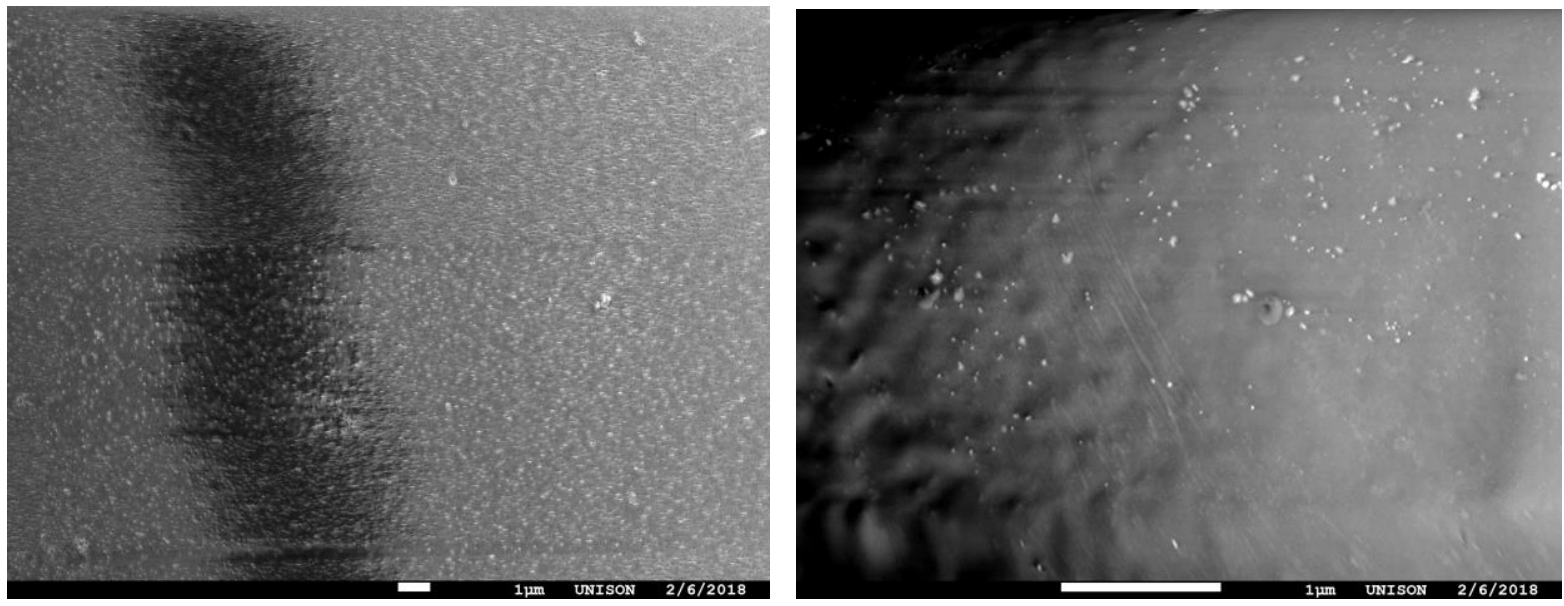

Figure 1. SEM images of CdTe quantum dots embedded in PMMA deposited over soda lime glass substrate.

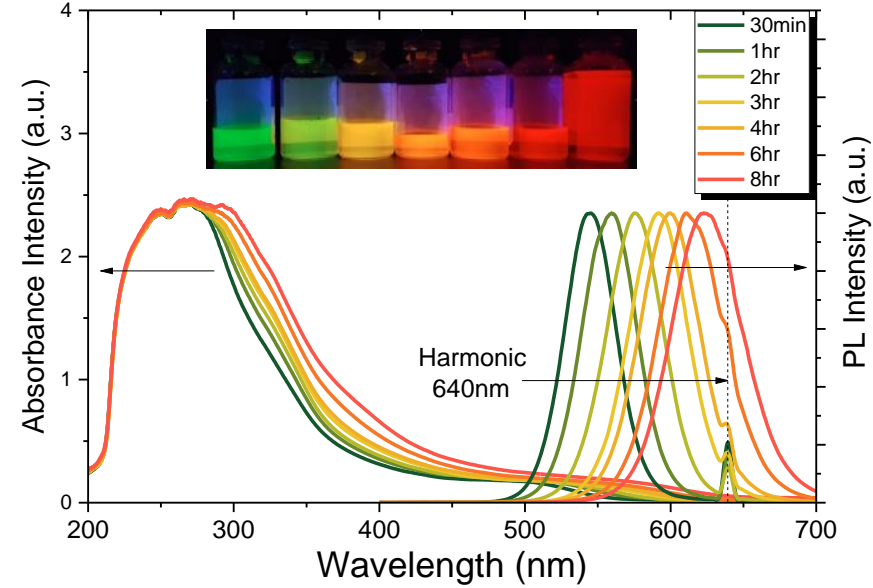

Figure 2. Absorbance and Photoluminescence of CdTe QDs.

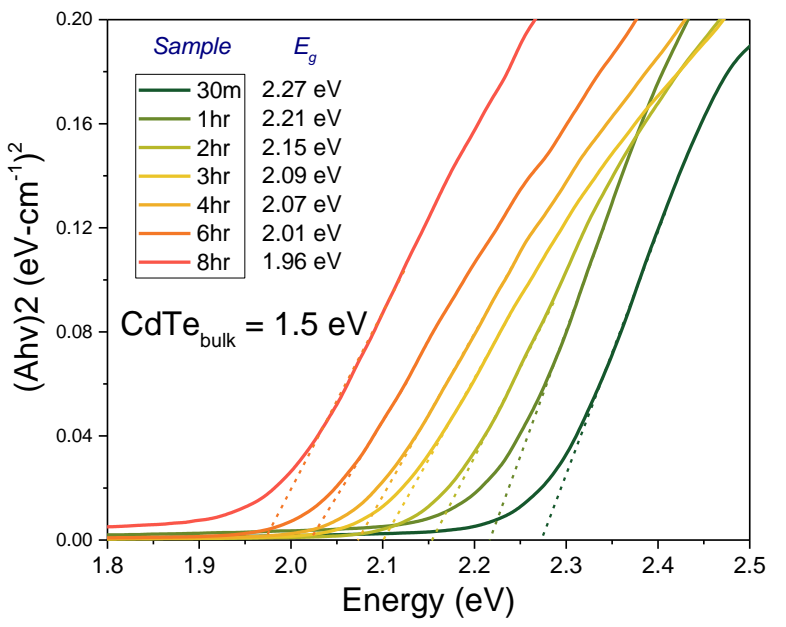

Figure 3. Band gap calculation using Tauc's law approximation. 